\title{
Vinculación con la Sociedad en la Universidad de Otavalo, Ecuador
}

\author{
Cecilia J. Polaino y Antonio de J. Romillo \\ Universidad de Otavalo, Cdla Imbaya, Ave. De los Sarances s/n y Pendoneros, Cantón Otavalo, \\ Provincia Imbabura, Ecuador. (e-mail: cpolaino@uotavalo.edu.ec; aromillo@uotavalo.edu.ec)
}

Recibido Oct. 11, 2016; Aceptado Dic. 16, 2016; Versión final Ene. 23, 2017, Publicado Jun. 2017

\begin{abstract}
Resumen
En este trabajo se presenta el Modelo de Vinculación con la Sociedad de la Universidad de Otavalo, creado con el objetivo de potenciar y mejorar la calidad de la gestión de esta función de vinculación a través de la realización de proyectos, prácticas pre-profesionales, capacitación y servicios profesionales de la universidad con el entorno. Se realizó una investigación documental sobre los modelos de gestión utilizados en otras universidades y empresas a través de los métodos de análisis, síntesis y modelación. Se definieron los ejes fundamentales que soportan y garantizan el funcionamiento del modelo, los criterios en que se basa, así como la identificación de los elementos que forman parte de las entradas, procesos y salidas del modelo. Finalmente, se incluye un análisis comparativo de los resultados de la implementación del modelo en el que se puede apreciar un incremento de los indicadores del trabajo de vinculación de la Universidad de Otavalo con la sociedad.
\end{abstract}

Palabras clave: universidad-sociedad; vinculación con el medio; gestión universitaria; funciones sustantivas

\section{Relationship with Society at the University of Otavalo, Ecuador}

\begin{abstract}
In this work the Relationship with Society Model of the University of Otavalo is presented. The model was created to enhance and improve the quality of management of this substantive function through the implementation of projects, pre -professional training and professional services training with the environment. Documentary research on management models used in other universities and companies was performed, through the methods of analysis, synthesis and modeling. The fundamental axes that support and guarantee the functioning of the model, the criteria on which it is based, as well as the identification of the elements that are part of the inputs, processes and outputs of the model are defined. Finally, a comparative analysis of the results of the implementation of the model is done, showing that there was an increase in work relationship indicators of the University of Otavalo with society.
\end{abstract}

Keywords: university-society; relation with the society; university management; substantive functions 


\section{INTRODUCCIÓN}

La extensión universitaria, que en el caso de Ecuador se ha conceptualizado como vinculación de la universidad con la sociedad (LOES 298, 2010), es una de las funciones sustantivas de la educación superior que, de conjunto con la formación académica y la investigación, tiene el propósito de solucionar problemas de la comunidad, a través de proyectos, prácticas pre-profesionales, capacitación y otros servicios profesionales, que posibilitan la comunicación y la interacción efectiva de la universidad con su entorno, así como la integración de la teoría con la práctica del quehacer universitario en función del desarrollo social sostenible Dicha vinculación constituye una tarea misional de la Universidad de Otavalo, que permite extender el saber universitario más allá de sus "fronteras" y colocarlas al servicio de la sociedad urbana y rural. Proceso en el que participan docentes y estudiantes en aras de ofrecer soluciones o paliativos a las diferentes problemáticas sociales, al brindar un servicio científico técnico desde la perspectiva de cada carrera y su campo de especialidad en una búsqueda permanente del mejoramiento de la calidad de vida de la sociedad.

La extensión universitaria, por su importancia se ha llegado a reconocer como la tercera misión de la educación superior (Loi y Di Guardo, 2015; DAN, 2012) con diferentes enfoques en cuanto a sus características y tendencias según el criterio de los tratadistas (Montesinos et al., 2008; Etzkowitz, 2014), pero con un criterio generalizado sobre su trascendencia en la proyección estratégica de la educación superior.

El proceso de formación de los estudiantes por medio del trabajo de vinculación está relacionado con el modo 2 de producción del conocimiento (Gibbons et al., 1997; Nowotny, et al., 2003 ), y complementa el modelo investigativo universitario de Humbold (Henningsen et al., 2013) lo que se logra a partir de una serie de consideraciones como, por ejemplo, la intención de ser útil a la sociedad, participar en un proceso donde el conocimiento y sus resultados se difundan a la sociedad a través de las habilidades y experiencias que aportan los que intervienen en ella y de que los aprendizajes estarán dados en función de la multiplicidad de ambientes, contextos, dimensiones y condiciones del entorno. Posición coincidente asume Osorio (2006) cuando señala que el modo 2 es un tipo de investigación “... caracterizada por un enfoque transdisciplinar, en procura de un conocimiento útil y negociado entre los actores académicos y sociales. Lo importante a tener en cuenta es que el modo 2 es una manera de acercar la industria, el medio ambiente y la sociedad, al trabajo investigativo bajo una concepción distinta".

Por su parte, Malagón (2006) señala que el conocimiento debe ser apropiado de diferentes maneras, dentro y fuera de la universidad ya que reconoce que la producción del conocimiento constituye una de las formas más claras de interacción entre la universidad y el contexto en que se inserta; mientras que en fecha más reciente, Larrea (2014) reconoce que la organización de las Instituciones de Educación Superior (IES) por dominios plantea la necesidad de generar una nueva organización académica diseñada desde el citado modo 2 del conocimiento con "vasos comunicantes" entre la formación, la investigación y la gestión social del conocimiento. Rodríguez-Ponce y Pedraja-Rejas (2016) marcan que el proceso final de la gestión del conocimiento es aplicarlo y al mismo tiempo convertirlo en productos valiosos para la organización (Osei-Bryson et al., 2014; Patel y Gorvadiya, 2014). Al mismo tiempo (Choi et al., 2010) indica que crear y compartir conocimiento es condición necesaria pero no suficiente para aplicar conocimiento con éxito.

La importancia de la vinculación universidad-empresa-gobierno ha sido distinguida por Vite (2009), citado por Bautista (2015), al identificarle como uno de los ejes de las IES donde se disputa el quehacer de la universidad en la generación, aplicación y transferencia del conocimiento

En otro orden, resulta válido acotar que la elaboración de modelos, tanto en el sector empresarial como en el educativo, se utiliza para organizar los procesos - de producción, de formación, de investigaciónde las instituciones, de manera que permitan la adecuada planificación, ejecución, control y evaluación de dichos procesos. Así, resulta habitual que se encuentren referencias teóricas (Abreu et. al., 2016; Muñoz, 2011; Mayorga y Madrid, 2010) en cuanto a distintas formulaciones de modelos que coadyuvan a una mejor comprensión de su utilización. Ya llevado al específico campo de la labor de vinculación, se puede apreciar la concepción de modelos de gestión como uno de los primeros pasos de la dirección universitaria en su trabajo de vinculación con la sociedad y convertirles en un proceso sistemático que permita planificar, organizar, controlar y evaluar el proceso de forma que provoque la implicación de profesores, estudiantes, agentes sociales y ahorro de recursos, con lo que se obtiene un provecho añadido en el entendido de formación de valores en todos los que intervienen en dicho proceso y de incidencia en el entorno a partir de la integración de las funciones sustantivas de la Universidad. 
Al respecto, Santana y Medina (2012), citando a Campos y Sánchez (2005) sostienen que corresponde a las IES: “... construir 'redes de acción' que tienen influencia más allá de la propia universidad; es decir, que incluyen un programa fuertemente relacionado con otros agentes, como el gobierno, las entidades productoras, el sistema educativo en su conjunto y sobre todo, los centros de investigación del nivel superior, e incluso, sectores de la sociedad que puedan colaborar en una estructura realmente operativa". A su turno, Vega-González (2011) indica que: "... un primer paso en la búsqueda de elementos que contribuyan a mejorar la percepción y a definir el valor intangible que aporta la vinculación y la gestión tecnológica de los proyectos patrocinados, es la construcción de un modelo empírico de gestión tecnológica de proyectos".

Sobre la base de las posiciones teóricas expuestas y en atención a las características propias de la institución y del contexto en que está ubicada, la Universidad de Otavalo ha desarrollado un modelo de vinculación con la sociedad, que integra los diferentes procesos universitarios, los actores principales, las diversas modalidades de actuación y su adecuada articulación con los sectores y ejes estratégicos de desarrollo social, cultural, ambiental y productivo de la zona a la que pertenece, con el propósito de lograr la máxima efectividad en el importante servicio que debe brindar toda institución de educación superior al desarrollo sostenible de la sociedad.

\section{ALCANCE DEL TRABAJO DE VINCULACIÓN}

El alcance del trabajo de vinculación con la sociedad tiene tres componentes fundamentales: capacitación, consultorías-prestación de servicios y la práctica pre-profesional (PPP), de conformidad a lo establecido en el Reglamento del Régimen Académico de la Universidad, suscrito por el Consejo Universitario de la Universidad de Otavalo en el 2015. En cualquiera de estos tres componentes puede ser conveniente la utilización de la figura del proyecto de vinculación como vía organizativa para la realización del trabajo, en tanto resulta prácticamente imprescindible en los componentes de las consultorías y prestación de servicios, con vistas a lograr la efectividad en la identificación, planificación, ejecución, monitoreo y evaluación de la actividad a realizar. En la figura 1 aparece el diagrama donde se han representado los campos de trabajo relativos a la vinculación con la sociedad y cada cuadro se encuentra bien definido en el Reglamento del Régimen Académico de la Universidad.

Para llevar a cabo el trabajo de vinculación con la sociedad, en la Universidad de Otavalo (UO) se han establecido las consideraciones siguientes: (1) Los docentes de tiempo completo deben realizar horas de trabajo en la vinculación, así como investigación, por lo que son los encargados de desarrollar y ejecutar los proyectos y/o acciones en cualquiera de sus tres componentes; (2) Cada carrera presentará proyectos anuales de vinculación con la posibilidad de extenderse en tiempo, de acuerdo con sus resultados, y la factibilidad de asociarse entre carreras para presentar proyectos interdisciplinares. (3) Las necesidades no contempladas en los planes estratégicos y operativos de la universidad y las carreras, pero que sean planteadas operativamente por las instituciones nacionales, regionales y locales, son también atendidas con prioridad y se abordan como extra plan en caso de existir posibilidades para ello; (4) La dirección de la carrera designa formalmente un coordinador de sus proyectos de vinculación, quien se encargará de su desarrollo y ejecución, con la participación de estudiantes durante todos los procesos del proyecto: identificación, diseño, ejecución y evaluación; (5) Las prácticas de servicio comunitario con la sociedad tienen una duración de -al menos- 160 horas para cada estudiante, de conformidad con el Reglamento del Régimen Académico; (6) Los estudiantes deberán presentar los documentos y certificados que acrediten el total de horas realizadas en prácticas de servicio comunitario y pre-profesional, para que se añadan en su carpeta en Secretaría Académica y encontrarse aptos para su graduación; y (7) Las actividades de vinculación se realizarán durante el semestre, los intersemestres o durante un periodo alternativo diferente, que coordine el docente con los estudiantes y las instituciones donde se realizan las prácticas.

La estructura organizativa (figura 2), aprobada por el Consejo Universitario de la institución, contempla la existencia de una Comisión de Vinculación, subordinada al Vicerrector Académico, que es la encargada de coordinar y dar seguimiento a las actividades de vinculación de la Universidad en su conjunto con la sociedad. Actividades que se realizan a través de proyectos y programas que beneficien a la colectividad, que deben estar incluidos en el plan operativo institucional y los proyectos articulados con los objetivos del Plan Nacional para el Buen Vivir (PNBV), así como en los planes de las agendas territoriales con las que se relacionen los proyectos. Oportuno aclarar que el PNBV constituye una especie de guía de la actuación pública en Ecuador para construir los derechos de las personas, a partir de la consideración de la naturaleza y los elementos propios del medio ambiente como connaturales al ser humano. Este plan está destinado a ser un referente en Latinoamérica y encuentra reflejo en la norma constitucional a través de la consagración de derechos fundamentales. 


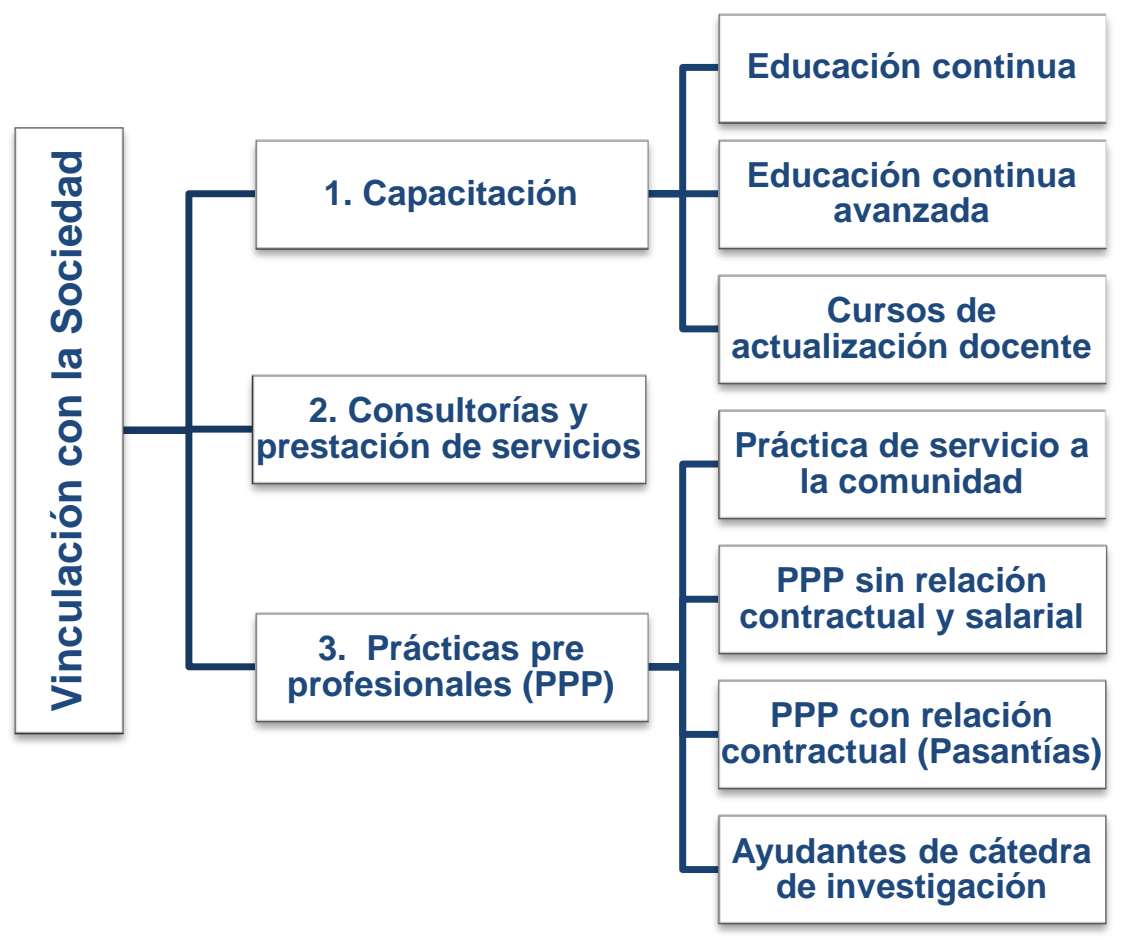

Fig.1: Diagrama del alcance del trabajo de Vinculación con la Sociedad

(Tomada de Reglamento de Régimen Académico de la Universidad de Otavalo, 2016a)

\section{MATERIALES Y MÉTODOS}

Para establecer las bases del modelo propuesto se realizó una investigación mediante la utilización del método de análisis y síntesis de diferentes documentos, en los que se encuentran definidos aspectos relativos al trabajo de vinculación con la sociedad y que aparecen en los artículos de los instrumentos jurídicos siguientes: (i) Constitución de la República del Ecuador, Arts. 27, 350, 387 y 388; (ii) Ley Orgánica de Educación Superior (LOES), Arts. 8, 13, 88, 107, 125, 127 y 160; (iii) Plan Nacional de Vinculación de la Educación Superior con la Sociedad; y, (iv) Reglamento del Régimen Académico del CES, Arts. 6 y 77.

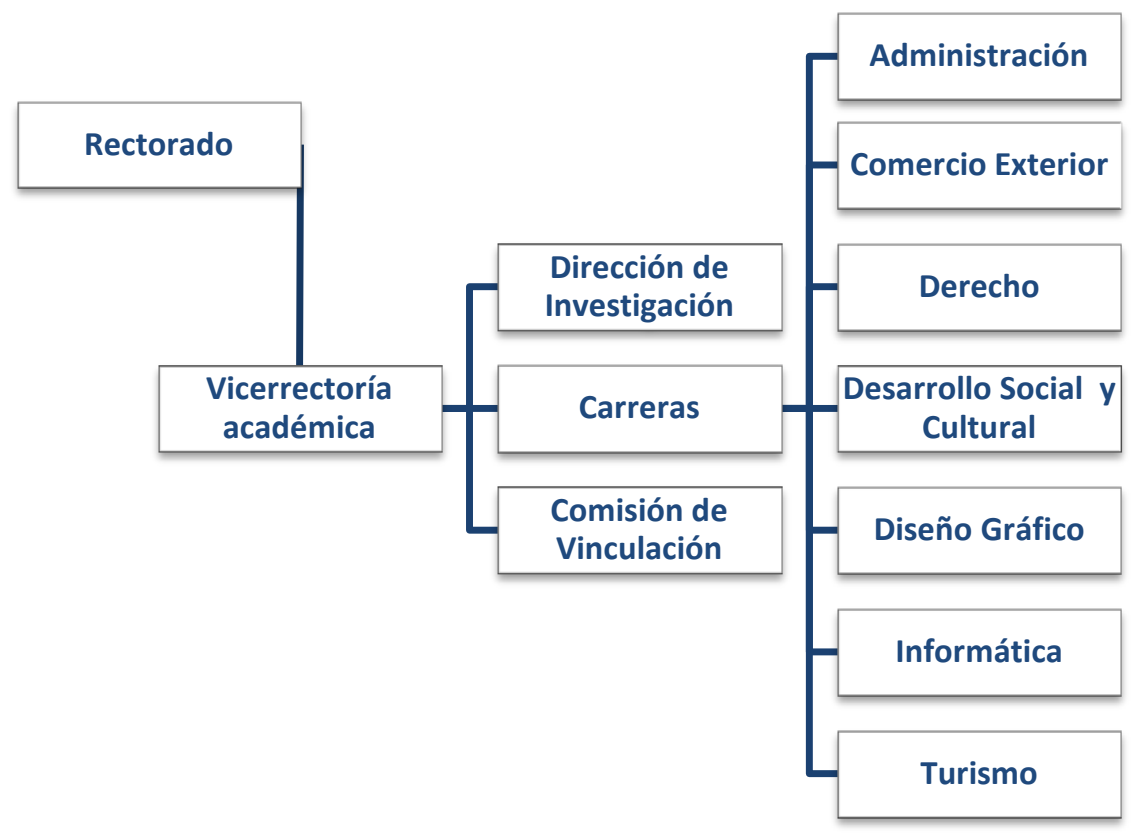

Fig. 2: Estructura jerárquico-organizativa de la Comisión de Vinculación con la Sociedad (Tomada de Estructura organizativa de la Universidad de Otavalo, UO, 2016b) 
De la misma forma se consideró, también, la necesaria prioridad de la actividad de vinculación con la sociedad en el plan estratégico de desarrollo institucional (PEDI 2015-2020), donde se describe como Misión y Visión lo siguiente: Misión Institucional: "Somos una institución intercultural autofinanciada de educación superior, que garantiza con elevada calidad la formación integral y continua de profesionales, a cuyos conocimientos científicos técnicos, se aúna la formación humanista, solidaria y creativa, que integrada al impacto de los procesos de investigación y vinculación con la sociedad, contribuye de forma decisiva al desarrollo sustentable local, regional y nacional"; Visión institucional: "Ser, para el año 2020, una universidad intercultural autofinanciada reconocida en el Ecuador por su compromiso con el buen vivir de los ecuatorianos, la calidad e integración de sus procesos de formación, investigación y vinculación con la sociedad y sus valores compartidos de capacidad creativa, responsabilidad, entrega emotiva, avance sustentable y relaciones solidarias".

Del propio documento se constata que la Vinculación con la Sociedad se encuentra entre las áreas de resultados claves de la UO, planteándose como estratégico el siguiente: Objetivo 5: "Incrementar los vínculos de la comunidad universitaria con su entorno, que posibiliten la integración e impacto de los procesos universitarios, en el desarrollo sustentable local, regional y nacional, así como una mayor calidad en el proceso de formación." De manera que esta conexión con el entorno social, como proceso de formación de la Universidad, se articula coherentemente en la planeación estratégica y la dirección por objetivos, lo que tributa -a partir del trabajo socio profesional universitario- a los valores compartidos de la Universidad. Pero, no se trata de un hecho aislado o casual, sino que encuentra sustento teórico en otros modelos de gestión aplicados a disímiles procesos sustantivos de instituciones académicas (González y González, 2006; Vizcaíno, 2013) y empresariales (EFQM, 2015; Álvarez, 2012), todos concebidos con el objetivo de buscar la excelencia en la gestión de los procesos de las entidades.

A partir del análisis de los modelos empleados en instituciones de educación superior, se denota que -en todos los casos- aparecen definidas las variables de entrada y salida, así como los procesos que se llevan a cabo; y, en correspondencia, el modelo que se presenta cumple con estos requisitos. Para evaluar los resultados en las salidas -entiéndase: personal capacitado, consultorías realizadas, estudiantes y profesores mejor preparados, fortalecimiento de la relación universidad-sociedad, impactos positivos a la sociedad y satisfacción de los clientes y usuarios- se diseñaron y aplicaron encuestas, así como se solicitaron avales a las organizaciones participantes.

\section{MODELO DE VINCULACIÓN DE LA UNIVERSIDAD DE OTAVALO}

Los criterios en que se basa la propuesta del presente trabajo están fundamentados en el modelo de la Fundación Europea para la Gestión de la Calidad (EFQM); fundación creadora y gestora del premio a la Excelencia, que reconoce la excelsitud en gestión en las organizaciones. En aras de garantizar la calidad de la gestión de vinculación en la UO, los aspectos a tener en cuenta son: satisfacción de las personas que intervienen en el grupo de procesos de vinculación con la sociedad; adecuada definición de dichos procesos; uso correcto de los recursos humanos y materiales que intervienen en las actividades; e impacto positivo del trabajo de vinculación, tanto inter como extra universitario.

Constituyen entradas al modelo los elementos siguientes: los recursos humanos de la universidad y la sociedad que intervienen en el grupo de procesos y que actúan de forma directa o indirecta; los recursos materiales, económicos, equipamiento y sistemas de información de ambas partes; las necesidades del entorno o escenarios laborales y sociales y las alianzas que se establezcan para el cumplimiento de los objetivos del PNBV, de los Gobiernos Autónomos Descentralizados (GADs) y de las agendas territoriales; los requisitos que se establecen en el currículo de las diferentes carreras para cumplir con la formación integral de los estudiantes; las políticas y estrategias establecidas en la universidad con relación a la investigación y a la vinculación, de acuerdo al Reglamento del Régimen Académico (RRA) y el cumplimiento de indicadores y estándares establecidos por los organismos de control de la educación superior, entre los cuales pueden citarse al Consejo de Educación Superior (CES), al Consejo de Evaluación, Acreditación y Aseguramiento de la Calidad de la Educación Superior (CEAACES) y la Secretaría Nacional de Educación Superior, Ciencia y Tecnología (SENESCYT).

Como grupo de procesos se establecen los relacionados con los tres componentes de la vinculación: capacitación, consultorías-prestación de servicios y la práctica pre-profesional en todos sus tipos y modalidades junto con los procesos de dirección de proyectos: identificación-diagnóstico, planificación, ejecución, seguimiento, control, cierre y evaluación de los impactos y satisfacción de los beneficiarios, de acuerdo a las metodologías establecidas y acordadas por las partes. Las salidas constituyen el elemento más importante pues serán las metas y resultados logrados en el proceso de vinculación universidad-sociedad y entre ellas se encuentran: personal capacitado, resultados o productos de los proyectos, consultorías y servicios técnicos, estudiantes y profesores mejor preparados, fortalecimiento de la relación universidad-sociedad, impactos positivos y una mayor contribución al desarrollo productivo, social y cultural del entorno, que constituye razón de ser de la Universidad. 
Para realizar los procesos mencionados, la Comisión de Vinculación ha desarrollado, socializado y aprobado, los instrumentos necesarios que garantizan su cumplimiento, como son: el programa de vinculación, definición de los temas a trabajar por las diferentes carreras en coordinación con el programa de investigación de la universidad, las guías para presentar los perfiles y los informes finales de los proyectos, el seguimiento y control de los proyectos, la evaluación de la satisfacción de los participantes, beneficiarios o partes interesadas, así como la evaluación de los resultados de los proyectos a través de indicadores evaluativos (eficiencia, eficacia, pertinencia e impacto). En la figura 3 se representa un esquema con las entradas, procesos y salidas del modelo propuesto e implementado en la Universidad de Otavalo.

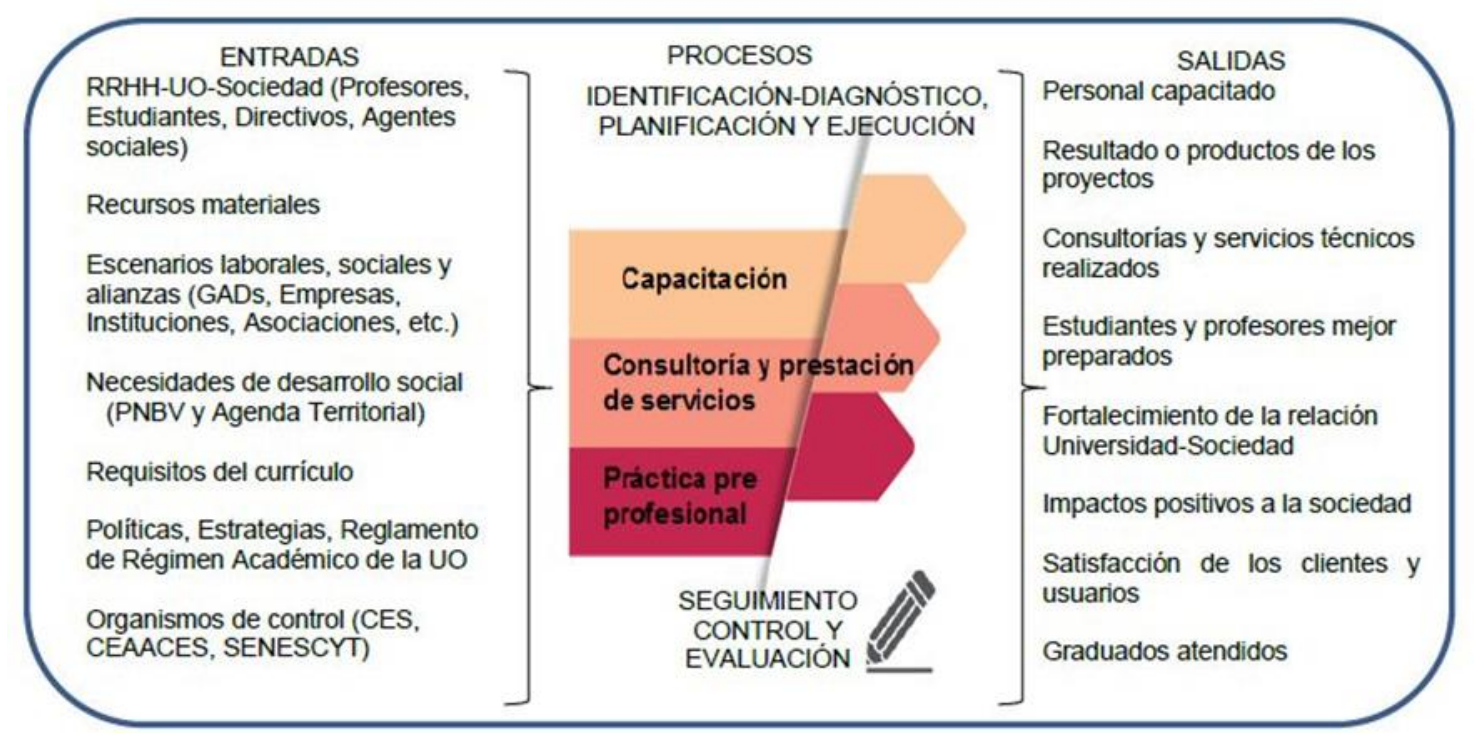

Fig. 3: Modelo de vinculación con la sociedad de la Universidad de Otavalo

En el estudio realizado sobre la sustentación teórica de los principios que soportan los diferentes modelos de extensión universitaria, se aprecia que muchas universidades extrapolan los principios y valores compartidos de la institución de educación superior a la extensión universitaria que realizan, sin diferenciar principios propios del proceso extensionista, sin embargo los autores que definen principios propios de modelos de los procesos de extensión universitaria, los contextualizan a los tipos, propósitos, objetivos y condiciones para los que ha sido concebido el modelo (Ortiz-Riada y Morales-Rubiano, 2011). Entre estos últimos se encuentra el modelo de extensión universitaria desarrollado para las instituciones de educación superior cubanas que determina como principios: participativo, dialógico, creativo y contextualizado (González y González, 2006), que por su carácter generalista requiere de su contextualización por las diferentes universidades que lo apliquen, tal y como se expresa en uno de dichos principios.

En el caso de la Universidad de Otavalo, la institución tiene establecidos los valores compartidos expresados en su Misión, que rigen los modos de conducta del quehacer universitario en su conjunto y que están presentes en sus procesos sustantivos, Enmarcados en dichos valores compartidos, teniendo en cuenta los propósitos, objetivos y contextualización del modelo de vinculación con la sociedad y como resultado de la investigación realizada, se determinaron los ejes fundamentales que soportan al modelo y garantizan su funcionamiento, los cuales son: Participación, Interacción, Creatividad, Solidaridad, Planificación, Conciencia, Flexibilidad, e Impacto Social. La validación de estos ejes se realizó en un taller que a tales efectos se realizó con el claustro de profesores de la universidad. En la Tabla 1 se describen Los ejes fundamentales del Modelo de Vinculación de la Sociedad de la Universidad de Otavalo.

\section{PROGRAMA DE VINCULACIÓN CON LA SOCIEDAD}

A partir de los principios o ejes fundamentales que soportan el Modelo de Vinculación con la Sociedad de la Universidad de Otavalo se establece el Programa de Vinculación denominado "Transferencia de conocimientos para fortalecer las capacidades y potencialidades de la población desde una perspectiva multicultural y de inclusión con una mayor calidad en el proceso de formación" que tributa a la línea de investigación "Desarrollo humano sustentable en la visión del buen vivir y sus relaciones con zonas geoculturales desde una perspectiva transdisciplinaria" y, al mismo tiempo, contribuye al logro del objetivo estratégico relacionado con el proceso sustantivo de Vinculación de "Incrementar los vínculos de la comunidad universitaria con su entorno, que posibiliten la integración e impacto de los procesos universitarios, en el desarrollo sustentable local, regional y nacional, así como, una mayor calidad en el proceso de formación". 
Tabla 1: Ejes fundamentales que soportan al modelo

\begin{tabular}{|c|c|}
\hline Ejes & Descripción \\
\hline Participación & $\begin{array}{l}\text { Involucra a estudiantes, docentes, tutores y trabajadores de la Universidad e interesados del } \\
\text { entorno social desde una posición activa y de transformación de la realidad. En el esquema } \\
\text { planteado y aprobado, los dos sectores encuentran las mejores condiciones para ejecutar los } \\
\text { proyectos con beneficio mutuo. }\end{array}$ \\
\hline Interacción & $\begin{array}{l}\text { Se establece un diálogo intercultural importante y necesario en los momentos actuales para } \\
\text { cambiar la matriz productiva y cultural. }\end{array}$ \\
\hline Creatividad & $\begin{array}{l}\text { A partir de las nuevas ideas y propuestas que se reflejan en los proyectos de acuerdo al contexto } \\
\text { donde se van a realizar. }\end{array}$ \\
\hline Solidaridad & $\begin{array}{l}\text { Con los sectores sociales que lo necesitan, especialmente los sectores considerados como } \\
\text { vulnerables y marginales. }\end{array}$ \\
\hline Planificación & $\begin{array}{l}\text { Basado en los planes estratégicos y operativos anuales de la institución y de la carrera, que se } \\
\text { complementa con los principios básicos del Buen Vivir contenidos en el Plan Nacional 2014-2017. }\end{array}$ \\
\hline Conciencia & $\begin{array}{l}\text { De los problemas que vive la sociedad. El elemento formador distingue a cada proyecto, pues } \\
\text { persiguen involucrar al estudiantado de forma real, proactiva y con un alto sentido ético del trabajo } \\
\text { compartido, en aras de convencerles de la relevancia de su desempeño en el radio de acción } \\
\text { universitario. }\end{array}$ \\
\hline Flexibilidad & De manera que sean tenidas en cuenta las especificidades de cada sector de la población. \\
\hline Impacto Social & $\begin{array}{l}\text { El fin máximo de la vinculación es dejar huella positiva en la población, región e instituciones en las } \\
\text { que se desarrolla. }\end{array}$ \\
\hline
\end{tabular}

Operativamente, la ejecución del programa de vinculación depende de la oferta académica de las diferentes carreras, así como de las necesidades de la comunidad y la disponibilidad logística y tecnológica con la que cuenten. Cada carrera tributa al programa con sus proyectos de forma que respondan y contribuyan al cumplimiento de los objetivos y políticas del PNBV 2013-2017, las agendas territoriales y los planes de desarrollo y ordenamiento territorial de los gobiernos autónomos descentralizados. Se han definido los temas de vinculación de cada carrera, de los que se nutre el programa de vinculación y la línea de investigación de la Universidad. Los temas de vinculación por carrera se muestran en la Tabla 2.

Tabla 2: Temas de vinculación por carrera

\begin{tabular}{|l|l|}
\hline \multicolumn{1}{|c|}{ Carreras } & \multicolumn{1}{c|}{ Temas } \\
\hline $\begin{array}{l}\text { Administración y desarrollo de } \\
\text { empresas }\end{array}$ & Organización y promoción de MIPYMES y emprendimientos. \\
\hline Comercio Exterior & $\begin{array}{l}\text { Generación y desarrollo de conocimientos y acciones encaminadas al mejoramiento } \\
\text { productivo, económico, financiero, educativo y social del sector público y privado. }\end{array}$ \\
\hline Derecho & Fortalecimiento de la justicia, la seguridad y los derechos humanos. \\
\hline Desarrollo Social y Cultural & Desarrollo social e intercultural del buen vivir en la zona Norte de Ecuador. \\
\hline Diseño gráfico & $\begin{array}{l}\text { Desarrollo de productos gráficos como alternativas que suplan las insuficiencias del } \\
\text { sector público y/o privado. }\end{array}$ \\
\hline Informática & $\begin{array}{l}\text { Desarrollo informático para el buen vivir en la zona norte de Ecuador (creación de } \\
\text { modelos informáticos, herramientas informáticas y plataformas virtuales). }\end{array}$ \\
\hline Turismo & $\begin{array}{l}\text { Desarrollo y fortalecimiento del sector turístico fundamentado en el PNBV para } \\
\text { potenciar los recursos naturales y culturales. }\end{array}$ \\
\hline Centro e Idiomas & $\begin{array}{l}\text { Cultura, promoción y difusión del Kichwa como idioma de la cultura ancestral, en } \\
\text { franco reconocimiento a la interculturalidad de la región en que se encuentra } \\
\text { enclavada la Universidad. }\end{array}$ \\
\hline
\end{tabular}

\section{RESULTADOS}

A partir del análisis de los informes finales de los proyectos de vinculación de la UO, elaborados por la Comisión de Vinculación de los años 2012 al 2016, se construyó el grafico de la Figura 4. En un análisis comparativo, se evidencia la influencia positiva de la implementación del modelo a partir del año 2015. En la Figura 4 se puede observar cómo ha sido el comportamiento positivo de algunos indicadores de los proyectos de vinculación en relación con la cantidad de proyectos, participantes (alumnos y profesores) y entidades con las que se han realizado los proyectos. 


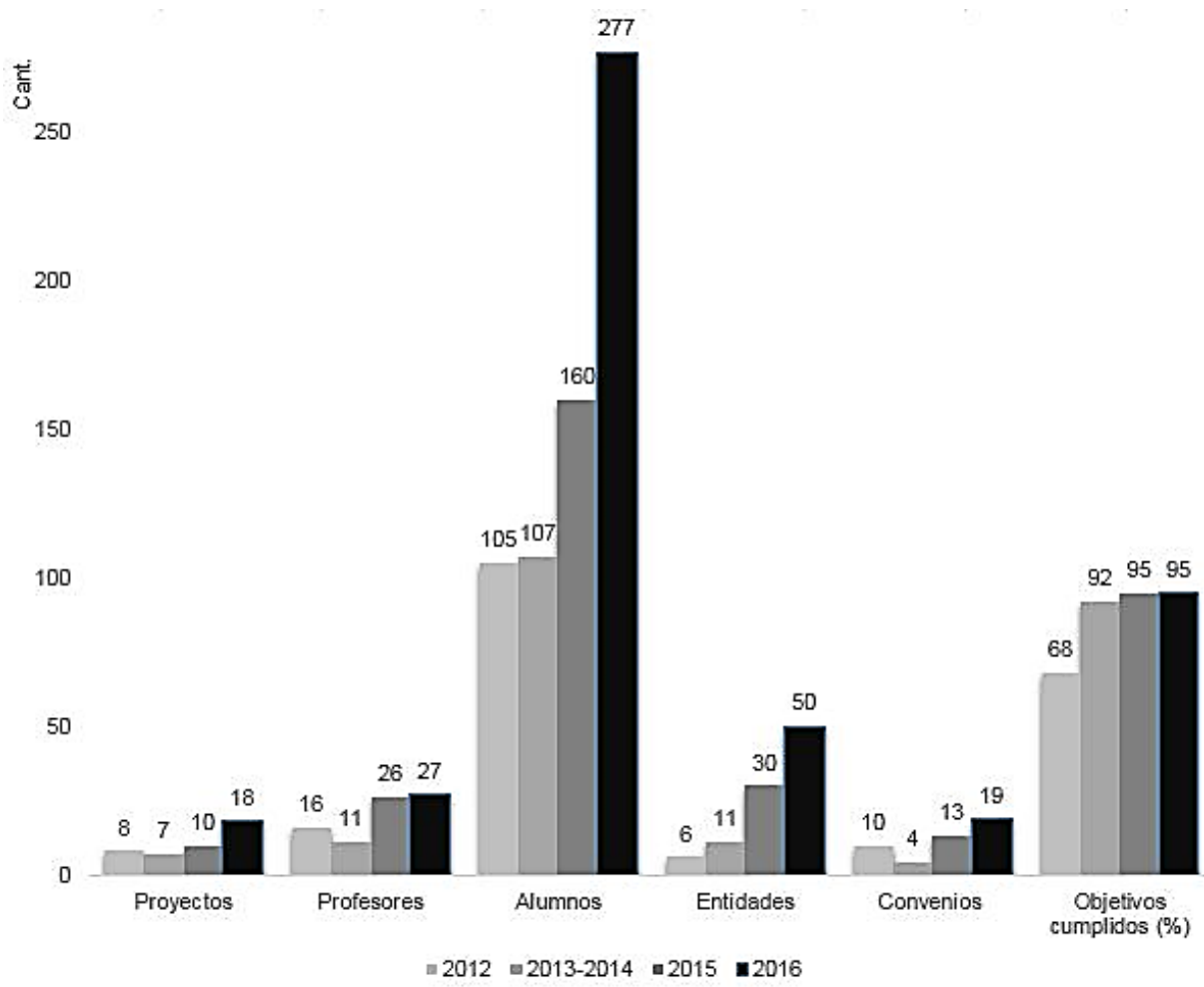

Fig. 4: Representación de indicadores de los proyectos en el período 2012-2016

La implementación del modelo fue a partir del mes de febrero de 2015 y en todos los aspectos, si comparamos con el año 2014, se denota el incremento en la cantidad de proyectos (7 a 18), profesores (de 11 a 27), alumnos (de 107 a 277) y de organizaciones o entidades relacionadas con los proyectos de vinculación (de 11 a 50). En el año 2016 se ejecutaron 18 proyectos y la participación de estudiantes, alumnos y organizaciones se ha acrecentado; por lo que se valida lo planteado en la literatura consultada sobre la utilización de modelos para organizar y optimizar los procesos de gestión. Aunque parezcan cifras modestas resulta necesario valorar que la Universidad de Otavalo es una institución de educación superior pequeña, en pleno desarrollo, que en la actualidad solo cuenta con 7 carreras universitarias, 38 profesores el $78 \%$ con cuarto nivel y $20 \%$ de PhD- y 450 estudiantes, por lo que -en consideración propia- los resultados logrados son verdaderamente significativos y van en ascenso, lo que constituye un claro indicador de progreso de la gestión universitaria. Como es de suponer, al tener un mayor número de proyectos realizados por año, existe un incremento en los recursos monetarios dedicados a los proyectos de vinculación cuya tendencia de crecimiento se observa con la línea discontinua en el gráfico de la Figura 5.

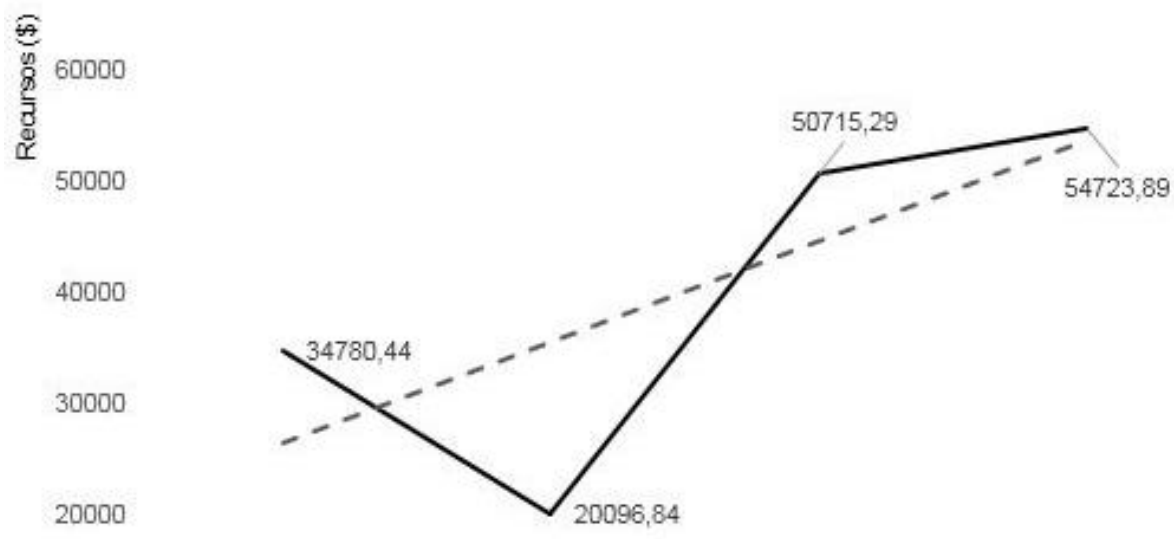

0

$2012 \quad 2015 \quad 2016$ Año

Fig. 5: Tendencia de los recursos utilizados en los proyectos de vinculación 
De una u otra manera en los diferentes proyectos realizados, de acuerdo a su tipología, se han obtenido los resultados que se plantean en las salidas del modelo, es decir: personal capacitado, consultorías realizadas, estudiantes y profesores mejor preparados, fortalecimiento de la relación universidad-sociedad, impactos positivos a la sociedad y satisfacción de los clientes y usuarios que han sido comprobados a través de encuestas y por los avales recibidos de la diferentes organizaciones participantes.

\section{CONCLUSIONES}

A partir de los resultados de la investigación se pueden presentar las conclusiones siguientes:

El modelo de vinculación de la Universidad de Otavalo -con sus entradas, procesos y salidas- contribuye al incremento de los vínculos de la comunidad universitaria con su entorno, lo que posibilita la integración e impacto de los procesos universitarios, en el desarrollo sustentable local y regional, así como una mayor calidad en el proceso de formación.

Los criterios en que se basa el modelo son: la satisfacción de las personas que intervienen en el proceso de vinculación con la sociedad; la adecuada definición de los procesos; el uso correcto de los recursos humanos y materiales que intervienen en las actividades; $y$, el impacto positivo que tiene el trabajo de vinculación universidad-sociedad, tanto inter como extra universitario.

Los procesos de dirección que lo conforman son: identificación-diagnóstico, planificación, ejecución, seguimiento, control, cierre y evaluación, todos relacionados con los tres componentes de la vinculación que resultan la capacitación, consultorías-prestación de servicios y la práctica pre-profesional en todos sus tipos y modalidades. Los principios o ejes fundamentales que soportan y garantizan el funcionamiento del modelo son: participación, interacción, creatividad, solidaridad, planificación, conciencia, flexibilidad e impacto social. El modelo de vinculación con la sociedad como proceso de formación de la Universidad se articula coherentemente en la planeación estratégica universitaria y la dirección por objetivos, lo que tributa a los valores compartidos de la Universidad, a partir del trabajo socio-profesional-universitario. Con la aplicación del Modelo desarrollado y el seguimiento de los procesos que le integran, se pudo evidenciar un incremento en la actividad de vinculación de la Universidad con la sociedad, avalado con los resultados alcanzados en cuanto a incremento de la cantidad de proyectos, alumnos, profesores, instituciones participantes y recursos asignados a los proyectos.

\section{REFERENCIAS}

Abreu, O., Naranjo, M.E., Rhea B., Gallegos, M., Modelo Didáctico para la Facultad de Ciencias Administrativas y Económicas de la Universidad Técnica del Norte en Ecuador, doi: 10.4067/S071850062016000400002, Formación Universitaria, 9(4), 3-10 (2016)

Álvarez, F. F., El Modelo EFQM y su impacto en la calidad y la excelencia (en línea), 3 de septiembre de 2015, https://goo.gl/6JF9Bi, (2012)

Asamblea Nacional de la República del Ecuador, Ley Orgánica de Educación Superior, https://goo.gl/S2L9BF, (en línea), 20 de diciembre de 2016, Asamblea Nacional, Registro Oficial Año II, Nº 298, 12 de octubre de 2010, Quito, Ecuador (2010)

Bautista, E. G., La importancia de la vinculación universidad-empresa-gobierno en México, https://goo.gl/oBMRN1, ISSN 2007-7467, Iberoamericana para la investigación y desarrollo educativo, 5(9), 1-2 (2014)

Campos, G. y Sánchez, G., La Vinculación Universitaria: Ese obscuro objeto del deseo, ISSN 1607-4041, Electrónica de Investigación Educativa, 7(2), 11-12 (2005)

Choi, S.Y., Lee, H. y Yoo, Y., The Impact of Information Technology and Transactive Memory Systems on Knowledge Sharing, Application, and Team Performance: A Field Study, MIS Quarterly, 34(4), 855-870 (2010)

Dan, M-C., The Third Mission of Universities in the Development Strategy of Vienna City, https://goo.gl/CIKSbR, ISSN 1453-1305, Informática Económica, 16(4), 49-56 (2012)

Fundación Europea para la Gestión de la Calidad (EFQM), ¿Qué es el modelo efqm? (en línea), https://goo.gl/T3UDDj, 3 de septiembre (2015) 
Henningsen, B., Schlaeger, J. y Tenorth, H-E., Humboldts Model: The Future of the Universities in the World of Research: Conference Report, $1^{\text {a }}$ edition, 201-231, BWV Verlag, Berlin, Germany (2013)

Gibbons, M., Limoges, C., Nowotny, H., Schwartzman, S., Scott, P., y Trow, M., The new production of knowledge. The dynamics of science and research in contemporary societies, $1^{\text {st }}$ ed., Pomores-Corredos S.A., Barcelona, Spain (1997)

Gómez, M. y Escamilla, C., Modelo de vinculación entre las Instituciones de Educación Superior y las empresas: gestión del conocimiento, https://goo.gl/7niKgX, ISSN: 0188-6266, Rev. Acta Universitaria, 22(2), $32-40(2011)$

González, M. y González, G., Extensión universitaria: Modelo teórico y de gestión para la educación superior cubana (en línea), 28 de agosto de 2015, https://goo.gl/0wMGZI, Ministerio de Educación Superior, Cuba (2006)

Etzkowitz, H., The entrepreneurial university wave: from ivory tower to Global Economic Engine, doi: 10.5367/ihe.2014.0211, Journal Industry and Higher Education, 28 (4), 223-232 (2014)

Larrea, E., Modelo de organización del conocimiento por dominios científicos, tecnológicos y humanísticos (en línea), 4 de mayo de 2015, https://goo.gl/9QA1Kg, Consejo de Educación Superior, Ecuador (2014)

Loi, M. y Di Guardo, M., The third mission of universities: An investigation of the espoused values, doi: 10.1093/scipol/scv012, J. Science and Public Policy, 42 (6), 855-870 (2015)

Mayorga M. J. y Madrid, D., Modelos didáctico y Estrategias de enseñanza en el Espacio Europeo de Educación Superior, http://www.tendenciaspedagogicas.com, ISSN 1989-8614, Tendencias Pedagógicas, 15(1), 91-111 (2010)

Montesinos, P., Carot, J.M., Martínez, J.M. y Mora, F., Third Mission Ranking for World Class Universities: Beyond Teaching and Research, doi:/abs/10.1080/03797720802254072, European Journal of Higher Education (EJHEJ), 33(2-3), 259-271 (2008)

Muñoz, C. M., Modelo de gestión para la vinculación Universidad-PyME en la República Argentina, Tesis de Magister en Administración de Negocios, Facultad Regional Buenos Aires, Universidad Tecnológica Nacional, Argentina (2011)

Nowotny, H., Scott, P. and Gibbons, M., Mode 2.The New Production of Knowledge, https://goo.gl/vCduRG, ISSN: 0026-4695, Minerva, 4(41), 179-194 (2003)

Ortiz-Riaga, M. C. y Morales-Rubiano, M. E., La extensión universitaria en América Latina: concepciones y tendencias, https://goo.gl/L31pAQ, ISSN 0123-1294, Rev. Educación y Educadores, 14(2), 349-366 (2011)

Osei-Bryson, K-M., Mansingh, G., Rao, L., Understanding and Applying Knowledge Management and Knowledge Management Systems in Developing Countries: Some Conceptual Foundations in Knowledge Management for Development, Springer Science +Business Media, 35, 1-15, doi: 10.1007/978-1-4899-73924_1, ISBN: 978-1-4899-7391-7 (Print) 978-1-4899-7392-4 (Online) New York, USA, 2014

Osorio, C., El modo 2 de investigación: la nueva forma de producción del conocimiento, http://hdl.handle.net/10893/6142, ISSN: 0121-0262, Heurística, 13, 7-8 (2006)

Patel, V.K. y Gorvadiya, A.B., Knowledge management: the need of modern organizations, Journal of Knowledge Management, 6(3), 259-261 (2014)

Rodríguez-Ponce, E. R. y Pedraja-Rejas, L. M., Percepciones sobre la Gestión del Conocimiento de Directivos Universitarios de Cuatro Universidades Chilenas, doi: 10.4067/S0718-50062016000400002, Formación Universitaria, 9(4), 41-52 (2016)

Vega-González, L. R., Modelo de gestión de proyectos de desarrollo tecnológico y vinculación de un centro de I\&DT universitario, ISSN 1405-7743, Ingeniería Investigación y Tecnología, 12(1), 73-82 (2011)

Vizcaíno, J., Diseño de un Modelo de gestión para la vinculación de las instituciones de educación, Tesis de Magister, Facultad de Ciencias Económicas, Universidad Central del Ecuador, Quito, Ecuador (2013) 